\title{
Verfassungsgericht kippt Drei-Monats-Frist zur Aufnahme der vertragsärztlichen Tätigkeit
}

—Das Bundesverfassungsgerichts hat am 26. September die Regelung aus $\$ 19$ Abs. 3 der Ärztezulassungsverordnung (Ärzte-ZV) für rechtswidrig erklärt (Az.: 1 BvR 1326/15). Bisher musste eine vertragsärztliche Tätigkeit innerhalb von drei Monaten aufgenommen werden ansonsten endete die Zulassung. Diese Vorschrift erklärten die Verfassungsrichter für nichtig, weil sie nicht von der Ermächtigungsgrundlage des $\$ 98$ SGB V gedeckt ist. In diesem Paragrafen wird das Bundesgesundheitsministerium berechtigt, die Zulassungsverordnung zu erlassen.

\section{MMW-KOMMENTAR}

Hintergrund des Urteils ist eine Verfassungsbeschwerde eines Medizinischen Versorgungszentrums (MVZ), dem eine Zulassung wegen Überschreitung der Drei-Monats-Frist verweigert wurde, was 2015 auch das Bundessozialgericht bestätigt hatte. Die Verfassungsrichter stellten nun klar, dass in das Grundrecht der Berufsfreiheit nach Art. 12 Abs. 1 GG nur auf gesetzlicher Grundlage und unter Beachtung des Grundsatzes der Verhältnismäßigkeit eingegriffen werden darf. Die in § 19 Abs. 3 Ärzte-ZV getroffene Regelung halte sich nicht an diesen Rahmen der parlamentarischen Ermächtigung.
Die Richter betonen, dass es sich bei dem Paragrafen aus der Zulassungsverordnung nicht um eine Vorschrift handelt, die lediglich "das Nähere" über die Teilnahme an der vertragsärztlichen Versorgung regelt. Vielmehr wird hier ein Beendigungstatbestand eingeführt, der in gesetzlichen, also vom Parlament geschaffenen Normen gar nicht vorkommt. Dies zeigt der Vergleich mit den im $S G B V$ geregelten Vorschriften. Diese enthalten für den Fall der Nichtaufnahme oder Nichtausübung der vertragsärztlichen Tätigkeit ein abgestuftes Regelungssystem, in welches sich die Bestimmung des $\S 19$ Abs. 3 Ärzte-ZV nicht einfügt.

\section{Bürokratie-Alarm bei Anstellung von Assistenten}

_ Einen Entlastungs- oder Sicherstellungsassistenten können Vertragsärzte nur mit Genehmigung der KV beschäftigen. Liegt eine solche nicht vor, riskiert der Praxisinhaber eine Kürzung des KVHonorars für die vom Assistenten erbrachten Kassenleistungen. Wenn eine vorsätzliche Handlungsweise vorliegt, kann sogar der Staatsanwalt ins Spiel kommen. Auch eine rückwirkende Genehmigung ist unzulässig. Dies geht aus einem Urteil des Sozialgerichts Marburg hervor (Az.: S 16 KA 531/13).

Im konkreten Fall wurde einer Hausärztin beschieden, dass sie die Leistungen ihrer Assistenten nur abrechnen dürfe, wenn die strengen formellen Grundlagen der Genehmigung nach $\$ 32$ Abs. 2 der Ärztezulassungsverordnung (Ärzte-ZV) erfüllt sind. Die Beschäftigung der Assistenten kann demnach nicht einfach auf einer

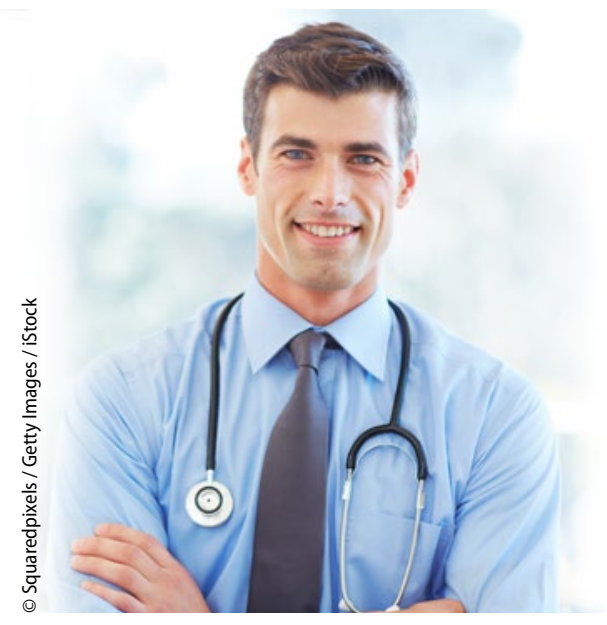

Er will Sie unterstützen - also erledigen Sie den Papierkram gewissenhaft!

Sammelerklärung angezeigt werden. Auch reicht die mündliche Auskunft eines KV-Mitarbeiters nicht als Genehmigung aus.

\section{MMW-KOMMENTAR}

Ein Praxisvertreter muss derselben Facharztgruppe angehören wie der Praxisinhaber. Eine Vertretung ist auch durch einen Weiterbildungsassistenten im letzten Weiterbildungsjahr möglich, die Vertretungszeit darf dann aber maximal 30 Arbeitstage (sechs Wochen) innerhalb von zwölf Monaten betragen. Wird dieser Zeitraum überschritten, muss die Beschäftigung eines Entlastungsassistenten durch die KV schriftlich bestätigt werden. Gleiches gilt für Vertretungen über einen längeren Zeitraum als drei Monate, auch wenn sie beispielsweise nur einen Tag in der Woche betreffen.

Dem Antrag, der spätestens drei Wochen vor Beginn der Vertretung gestellt werden muss, sollten Approbations- und Facharzturkunde des Assistenten beigefügt werden. Der Antrag muss laut § 32 Ärzte-ZV begründet werden und auch die voraussichtliche Dauer der Vertretung beinhalten. 